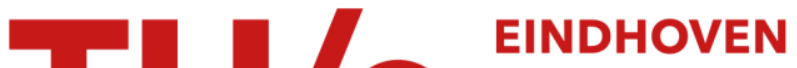 \\ UNIVERSITY OF \\ TECHNOLOGY
}

\section{Anisotropic Corbino magnetothermopower in a quantum Hall system}

Citation for published version (APA):

Zalinge, van, H., Heijden, van der, R. W., \& Wolter, J. H. (2003). Anisotropic Corbino magnetothermopower in a quantum Hall system. Physical Review B, 67(16), 165311-1/5. [165311].

https://doi.org/10.1103/PhysRevB.67.165311

DOI:

10.1103/PhysRevB.67.165311

Document status and date:

Published: 01/01/2003

\section{Document Version:}

Publisher's PDF, also known as Version of Record (includes final page, issue and volume numbers)

\section{Please check the document version of this publication:}

- A submitted manuscript is the version of the article upon submission and before peer-review. There can be important differences between the submitted version and the official published version of record. People interested in the research are advised to contact the author for the final version of the publication, or visit the $\mathrm{DOI}$ to the publisher's website.

- The final author version and the galley proof are versions of the publication after peer review.

- The final published version features the final layout of the paper including the volume, issue and page numbers.

Link to publication

\section{General rights}

Copyright and moral rights for the publications made accessible in the public portal are retained by the authors and/or other copyright owners and it is a condition of accessing publications that users recognise and abide by the legal requirements associated with these rights.

- Users may download and print one copy of any publication from the public portal for the purpose of private study or research.

- You may not further distribute the material or use it for any profit-making activity or commercial gain

- You may freely distribute the URL identifying the publication in the public portal.

If the publication is distributed under the terms of Article 25fa of the Dutch Copyright Act, indicated by the "Taverne" license above, please follow below link for the End User Agreement:

www.tue.nl/taverne

Take down policy

If you believe that this document breaches copyright please contact us at:

openaccess@tue.nl

providing details and we will investigate your claim. 


\title{
Anisotropic Corbino magnetothermopower in a quantum Hall system
}

\author{
H. van Zalinge, R. W. van der Heijden, and J. H. Wolter \\ COBRA Inter-University Research Institute, Department of Applied Physics, Eindhoven University of Technology, \\ P.O. Box 513, NL-5600 MB Eindhoven, The Netherlands
}

(Received 5 February 2002; published 16 April 2003)

\begin{abstract}
A Corbino-geometry contact configuration combined with a scanning laserspot as a heating source, is used for a thermovoltage mapping in a GaAs-AlGaAs quantum Hall device. For an isotropic system, the Corbino thermopower yields the diagonal component $\epsilon_{x x}$ of the thermoelectric tensor, which should be zero under the prevailing condition of phonon drag. The experiments reveal that $\epsilon_{x x}$ is large and anisotropic with respect to the crystallographic directions. The observations yield conclusive evidence that inhomogeneities are the origin for the existence of $\epsilon_{x x}$.

DOI: 10.1103/PhysRevB.67.165311

PACS number(s): 73.43.Fj, 73.50.Jt, 73.50.Lw
\end{abstract}

The transverse magnetothermopower or NernstEttingshausen (NE) effect, is the most poorly understood transport property of a two-dimensional electron gas (2DEG) in the quantum hall $(\mathrm{QH})$-effect regime. ${ }^{1-3}$ In the earliest investigations, ${ }^{4}$ it was found that the experimental magnetothermopower was orders of magnitude larger than expected for electron diffusion. The explanation in terms of phonon drag is satisfactory for the longitudinal component $S_{x x}$ of the thermopower tensor $\overleftrightarrow{S}$, but not for the NE component $S_{x y}$. The phonon-drag contribution to $S_{x y}$ theoretically is nearly zero, ${ }^{2}$ whereas experimentally $S_{x y}$ is as large as $S_{x x}$. A key experimental discovery to this problem, was the derivative relation $S_{x y}=\alpha B d S_{x x} / d B$ ( $\alpha$ a constant and $B$ magnetic field) by Tieke et al. ${ }^{1}$ An explanation was offered based on the presence of small lateral density variations, ${ }^{5}$ which implies that the macroscopically measured $S_{x y}$ is strongly intermixed with $S_{x x}$. An analogous experimental ${ }^{6}$ and theoretical $^{7}$ case exists for the resistivities.

In the work presented here, direct evidence is reported that inhomogeneities associated with molecular-beam epitaxial (MBE) growth have a profound effect on the thermopower. A scanning-heater measurement technique combined with a circular symmetric or Corbino-electrodes arrangement is developed. The Corbino arrangement is exceptionally well suited to tackle the problem, since for an ideal homogeneous sample the thermopower exclusively depends on a transport parameter that is zero for phonon-drag. Although the Corbino thermopower effect is known for half a century, ${ }^{8}$ surprisingly little use has ever been made of it, not only in two-dimensional (2D) systems, but even in 3D materials also. We are only aware of one recent work on bulk InSb, where the "geometric magnetothermopower" was investigated in a two-carrier system. ${ }^{9}$

The equation that governs the local electrical transport in a $2 \mathrm{DEG}$ is

$$
\mathbf{J}=\stackrel{\leftrightarrow}{\sigma} \mathbf{E}-\overleftrightarrow{\epsilon} \nabla \mathbf{T}
$$

where $\mathbf{J}$ is the electrical current density, $\mathbf{E}$ is the electrical field, and $\boldsymbol{\nabla} \mathbf{T}$ is the temperature gradient. The conductivity tensor $\stackrel{\leftrightarrow}{\sigma}$ and the thermoelectric tensor $\overleftrightarrow{\boldsymbol{\epsilon}}$ are the fundamental quantities that appear in a microscopic theory. The measured thermopower $\overleftrightarrow{S}$, defined by $\mathbf{E}=\overleftrightarrow{S} \nabla \mathbf{T}$, depends on the ex- perimental arrangement. In all experiments thus far, a Hall bar was used with $\mathbf{J}=0$, so that $\overleftrightarrow{S}=\overleftrightarrow{\sigma}^{-1} \overleftrightarrow{\epsilon}$. The measured components $S_{x x}$ and $S_{x y}$ of $\overleftrightarrow{S}$ both consist of an admixture of the components of $\overleftrightarrow{\sigma}$ and $\overleftrightarrow{\epsilon}$, which obscures the information on $\overleftrightarrow{\epsilon}$.

For an experiment with a Corbino-electrode arrangement and isotropic material, the condition of zero current can be imposed only in radial direction. In addition, azimuthal electric fields and temperature gradients cannot exist. Defining the Corbino thermopower $S_{c o r}$ as the ratio between radial electric field and radial temperature gradient gives

$$
S_{c o r}=\frac{\epsilon_{x x}}{\sigma_{x x}} .
$$

In the microscopic theory for phonon-drag, it follows that $\epsilon_{x x}$ is equal to zero ${ }^{2}$ and for sufficiently small resistivity $S_{x y}$ is practically zero as well. The simple dependence of $S_{c o r}$ on the fundamental quantities is appealing, in particular because it is straightforward to measure $\sigma_{x x}$ directly in the same sample in the same experiment.

A 4- $\mu \mathrm{m}$ GaAs bufferlayer was grown by MBE on a (001) GaAs substrate at approximately $620^{\circ} \mathrm{C}$. This was followed by a 20-nm layer of undoped $\mathrm{Al}_{0.33} \mathrm{Ga}_{0.67} \mathrm{As}$ at $630^{\circ} \mathrm{C}$; a 38-nm $\mathrm{Al}_{0.33} \mathrm{Ga}_{0.67} \mathrm{As}$ layer, $n$-type doped at 1.33 $\times 10^{18} \mathrm{~cm}^{-3}$; and finally, a $17-\mathrm{nm}$ GaAs cap layer. Standard Hall-bar characterization measurements yielded an electron density after illumination of $6 \times 10^{15} \mathrm{~m}^{-2}$ with a mobility of $\mu \sim 50 \mathrm{~m}^{2} / \mathrm{Vs}$. Square samples of $\sim 4 \times 4 \mathrm{~mm}^{2}$ were cleaved from the wafer. Ohmic contacts in a Corbino configuration, with inner and outer contact diameters of 1.2 and $2.0 \mathrm{~mm}$, were made by annealing a $210-\mathrm{nm}$-thick Au-Ge-Ni film for $60 \mathrm{~s}$ at $400^{\circ} \mathrm{C}$ [see inset Fig. 1(b)]. The samples were immersed in liquid helium at $1.5 \mathrm{~K}$ in a 7-T-magnet cryostat with optical windows. A $25-\mu \mathrm{m}$-diameter spot from a diodelaser at $630 \mathrm{~nm}$ wavelength could be scanned across the sample and the voltage induced in the contacts was recorded as a function of position. The laser was modulated at $70 \mathrm{~Hz}$ to allow the use of lock-in techniques. The thermal penetration depth $\delta_{t h}$ during a modulation period $1 / \omega$ is estimated using $\delta_{t h}=\sqrt{2 \alpha / \omega}$ with $\alpha=K / \rho C$ the thermal diffusivity of GaAs ( $K$ thermal conductivity, $\rho$ mass density, and $C$ specific heat). Using typical values of $\rho C=10 \mathrm{~J} / \mathrm{Km}^{3}$ at $1.5 \mathrm{~K}$, 


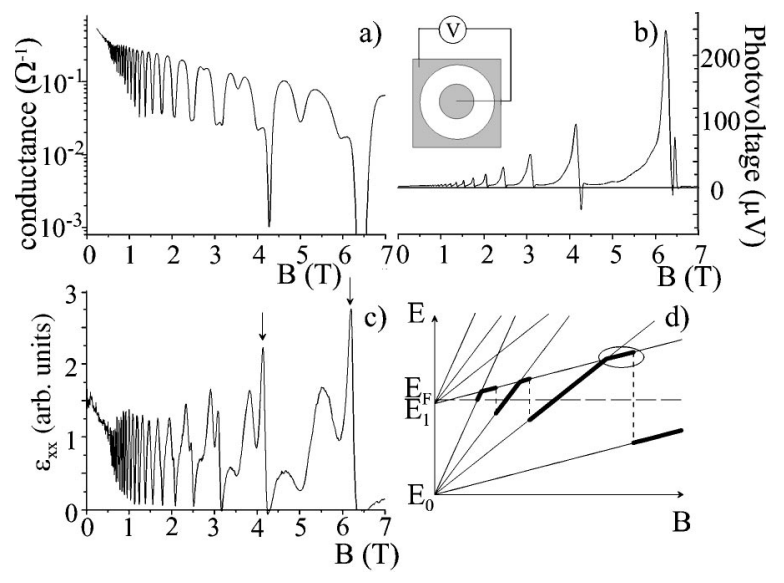

FIG. 1. (a) Magnetic-field dependence of two terminal conductance. (b) Photovoltage as a function of the magnetic field with laser directed at inner contact. This should correspond to $S_{c o r}$. The inset shows the used experimental configuration. (c) Magnetic-field dependence of $\epsilon_{x x}$, generated by multiplying the data from (a) and (b). The arrows indicate structure enhanced by the second subband. In (d), the influence of the population of the second subband is schematically shown.

(Ref. 10) and $K \sim 10 \mathrm{~W} / \mathrm{mK}$, (Ref. 11) $\delta_{t h}$ is found to be $\sim 5 \mathrm{~cm}$, or at least an order of magnitude larger than the sample dimension $(\sim 4 \mathrm{~mm})$. This means that the experiment represents essentially steady-state conditions so that all transients can be neglected in the analysis.

In Fig. 1(a), the measured Corbino conductance, which should be proportional to $\sigma_{x x}$, is given as a function of magnetic field. The shoulder at the high field side of a conductance peak occurs when the second subband is populated ${ }^{12}$ [see Fig. 1(d)]. The zero field second subband occupation is in the order of $10 \%$. For Fig. 1(b), the laser was directed at the center of the inner contact. The metal film is not transparent, so that the laser acts as a simple heater. The induced voltage in Fig. 1(b) should be proportional to $S_{\text {cor }}$. Note that it tends to diverge near the QH minima, in strong contrast with both $S_{x x}$ and $S_{x y}$ which are zero there. ${ }^{4}$ It should be noted that the expressions for the latter contain denominators that are always limited by the finite $\sigma_{x y}$, whereas $S_{c o r}$ is inversely proportional to $\sigma_{x x}$, which approaches zero in the $\mathrm{QH}$ regime. Assuming the validity of Eq. (2), the field dependence of $\epsilon_{x x}$ is generated from Figs. 1(a) and 1(b), and plotted in Fig. 1(c). Most remarkable is the prominence of the second subband in the thus generated apparent $\epsilon_{x x}(B)$ plot, indicated by arrows in Fig. 1(c).

In Fig. 2(a) the thermovoltage is plotted as the laserspot is moved across the electrode gap from position $\mathrm{x}_{1}$ to $\mathrm{x}_{2}$ as indicated schematically in Fig. 2(d). From a linescan as in Fig. 2(a), it is seen that the signal decays within about 0.1 $\mathrm{mm}$ from the contacts. It follows that the dominant signal is distinctly different from the one caused by carrier generation as observed in Hall bars. ${ }^{13,14}$ As follows from the data in Fig. 1(b), with the laserspot on the central contact and 2(a), with the laser scanned across the contact edge, strong signals are obtained both by illuminating the nontransparent metal contact as well as by shining directly into the semiconductor.
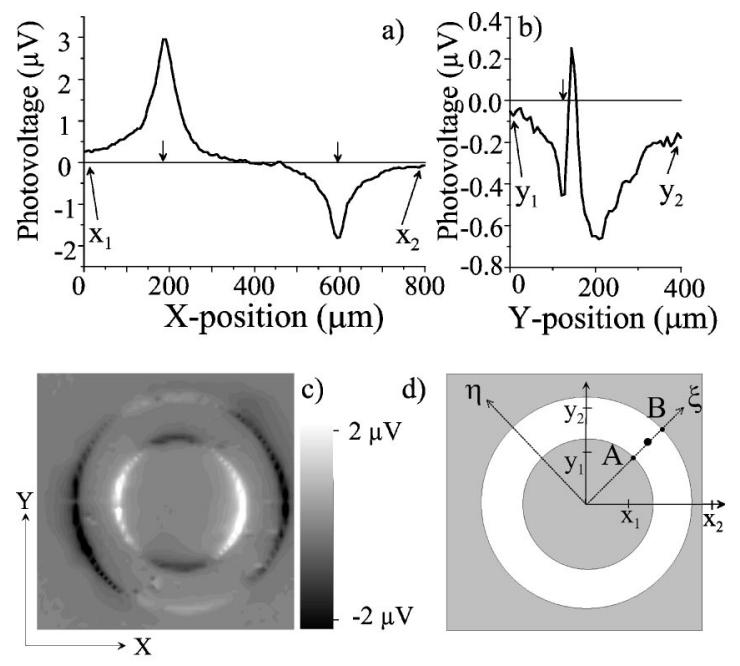

FIG. 2. (a) Linescan from $\mathrm{x}_{1}$ to $\mathrm{x}_{2}$ in (d) at $B=1.9 \mathrm{~T}$, when the laserspot is moved. (b) Linescan near the inner contact from $y_{1}$ to $\mathrm{y}_{2}$ at $5.0 \mathrm{~T}$. Arrows in (a) and (b) indicate 2DEG-contact boundary. (c) Photovoltage, when the laser is scanned over the sample at B = $1.9 \mathrm{~T}$. The sides of the square are parallel to the cleavage planes

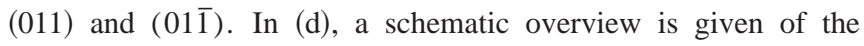
sample. The gives the position of the laserspot.

Hence, it is concluded that the signal is entirely dominated by thermal effects, even when the semiconducting region between the contacts is irradiated. A strictly contact-related effect, showing up only when the laserspot is positioned very close to the contact edge, can be present as well. It is observed at some parts of the contact and can be identified as a sharp feature superimposed on a broad structure. An example is shown in Fig. 2(b), where the laser is scanned across a contact edge, from $y_{1}$ to $y_{2}$ in the sketch of Fig. 2(d). The contact-related effect can be clearly distinguished from the thermal effect by both the width and the sign.

The sample was cooled over its entire surface, rather than on a circular edge. An estimate for the linewidth of the thermal signal in Fig. 2(a) may be obtained from a twodimensional cooling fin model, where the characteristic length is given by $L_{c f}=\sqrt{K D / 2 H}$ (Ref. 15), with $D$ the sample thickness $(0.4 \mathrm{~mm})$ and $H$ the surface heat transfer coefficient. Taking as an order of magnitude estimate for $H$ the inverse Kapitza resistance between a solid and liquid helium at $1.5 \mathrm{~K}$, one obtains $H \sim 3 \times 10^{3} \mathrm{~W} / \mathrm{Km}^{2}$ (Ref. 16) yielding $L_{c f} \sim 0.8 \mathrm{~mm}$. The experimental value of $\sim 0.1 \mathrm{~mm}$ [see Fig. 2(a)] is actually smaller than $D$, so that a $2 \mathrm{D}$ model will only yield an upper limit. Because $L_{c f}$ is much smaller than the lateral sample dimensions, the square sample layout does not affect the temperature gradient. In strong contrast to the Hall-bar data, ${ }^{14}$ the linewidth depends only weakly on the magnetic field for fields above $1 \mathrm{~T}$. This is in agreement with the thermal origin of the signal, since the temperature gradient is determined by the lattice thermal conductivity of the substrate and so is independent of $B$.

As seen from Figs. 2(a) and 2(b), the linescans in two perpendicular directions are very dissimilar. A complete overview is obtained by scanning the laserspot across the whole sample and representing the photovoltage at each 
point in a greyscale image as shown in Fig. 2(c). The most striking observation is the extreme anisotropy. Not only the signal strength in the two directions [01 $\overline{1}]$ and [011] differs by more than an order of magnitude, even the sign can be different. It was verified that this observation is not an artifact of the setup as the anisotropy direction remained fixed to the sample after it was mounted under a different angle in the cryostat.

As a consequence of the anisotropy as found using the scanning mode, the interpretation of the signal of a centrally heated Corbino [Figs. 1(b) and 1(c)] is no longer clear. Therefore, the case will be analyzed with the spot at an arbitrary position in the annular strip between the contacts [heavy spot in Fig. 2(d)]. Equation (1) can be worked out in a $(\xi, \eta)$ coordinate frame with the origin at the Corbinocenter and the $\xi$ axis in the radial direction passing through the spot, see Fig. 2(d). For a strictly isotropic medium, both $E_{\eta}$ and $\partial T / \partial \eta$ must be zero for every point along the $\xi$-axis. Integrating $E_{\xi}$ along the radial between the contacts from point $A$ to $B$ in Fig. 2(d) yields $\Delta V=S_{c o r} \Delta T_{\text {rad }}$, where $\Delta T_{\text {rad }}$ is the temperature difference between the contact boundaries. As a result, the interpretation of the image for an isotropic medium would be straightforward.

For anisotropic media, both $E_{\eta}$ and $\partial T / \partial \eta$ will still be zero for symmetry reasons for all points on a $\xi$ axis along symmetry directions. The analysis above still applies, and we have $\Delta V=S_{c o r} \Delta T_{r a d}$, with $S_{c o r}=\epsilon_{\xi \xi} / \sigma_{\xi \xi}$ and now the direction $\xi$ refers to a symmetry direction. Since $\nabla T$ is determined by the substrate, it is expected to be isotropic, or at least the same in the two equivalent bulk directions [011] and [011]. Therefore, the data in the image along the directions [01̄] and [011] can be directly interpreted as $\epsilon_{i i} / \sigma_{i i}$ data, where the index i stands for [011]] or [011]. Interpolation expressions for arbitrary directions between [011] and [011] will not be pursued in this paper. For the anisotropic case, the requirement of no external current, necessary for Eq. (2), is not rigorously fulfilled, because of shunting effects by the sample itself. Ignoring the shunting effects is reasonable, because the shunting impedance is of the order of the relevant intrinsic impedance.

With the heater spot centered at the central electrode as in Fig. 1(b), all directional information is lost. The measured thermopower will correspond to the one in the direction in which it is largest. A better arrangement is to use a contact configuration that does not strongly distort the thermovoltage distribution generated around a heated spot. Such an arrangement is provided by the segmented outer ring, shown in Fig. 3 (a). By selecting the proper electrodes on the outer ring, it is possible to measure directly the variation with magnetic field of $S_{c o r}$ in the [011] and [011] symmetry directions. Here, again, it is assumed that $\boldsymbol{\nabla T}$ does not depend on the magnetic field. The results are displayed in Fig. 3(b) and are consistent with the image of Fig. 2(c). The signal magnitude and even the sign are very different in the two directions. The data confirm in an independent way the extreme anisotropy of the magnetothermopower in the (001) plane.

To have another comparison of the present technique with the conventional methods for obtaining the thermopower, the
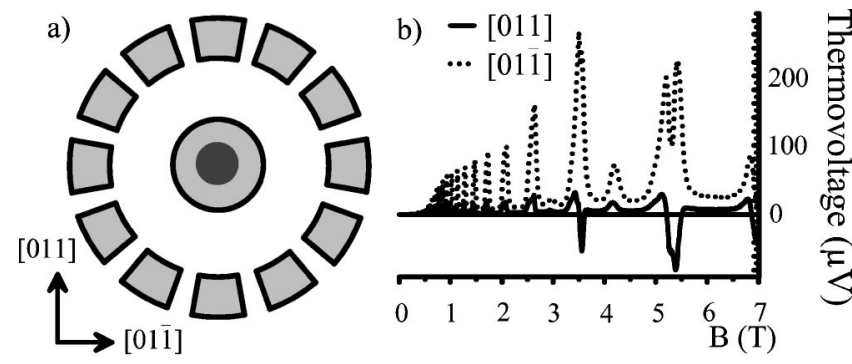

FIG. 3. (a) Electrode layout for the directional thermopower measurement. (b) Magnetothermovoltage obtained in the [011] and [011] direction using the configuration of (a).

evolution of a linescan was investigated in detail when the magnetic field was varied around a $\mathrm{QH}$ minimum. Here, the two-electrode configuration of Fig. 2 was used again. Figs. 4(b)-4(d) displays three linescans in the faint-signal (Y) direction of Fig. 2(c) for three characteristic fields near the $\mathrm{QH}$ minimum, with occupation number $\nu=4$ at $6.3 \mathrm{~T}$ [indicated by arrows in Fig. 4(a)]. The most remarkable phenomenon here is that for the lower and higher field values [Figs. 4(b) and 4(d)], the maximum and minimum in the thermovoltage are exchanged. At the very $\mathrm{QH}$ minimum [Fig. 4(c)], the signal behaves erratic. This might result from the extremely steep field dependence [see Fig. 1(b)]. A sign change for fields at opposite sides of a $\mathrm{QH}$ minimum is expected for $\epsilon_{x x}$ in the diffusion-dominated condition, and through this also in $S_{x y}$ in a Hall-bar configuration. ${ }^{17}$ A sign-change in the latter was observed also under phonon-drag conditions. ${ }^{1,4}$ In the strong-signal direction $(\mathrm{X})$ in the Corbino geometry, no sign change has been observed.

Some anisotropy of both thermopower and heat conductivity attributed to phonon focussing has been reported before. ${ }^{18}$ Phonon focusing in the (100) plane has fourfold symmetry, in contrast to Fig. 2(c), and can thus be excluded as a cause. For the same reason, a possible influence of the square sample layout can be ruled out, as this would lead to
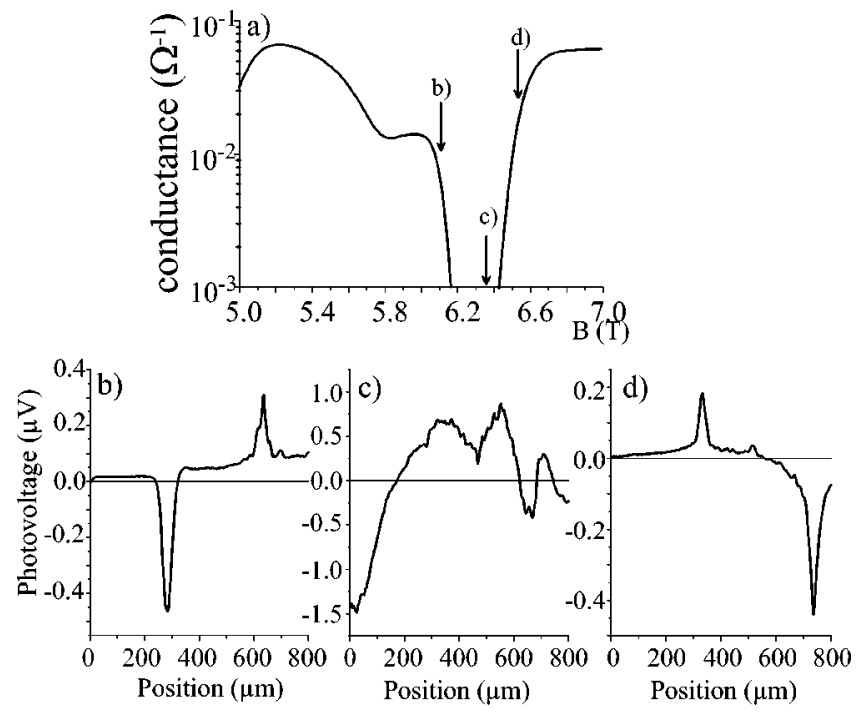

FIG. 4. (a) Part of $\sigma_{x x}$ data near 6.3 T. (b), (c), and (d) Linescans in the $Y$ direction of Fig. 1 (c) for fields as indicated in (a). 


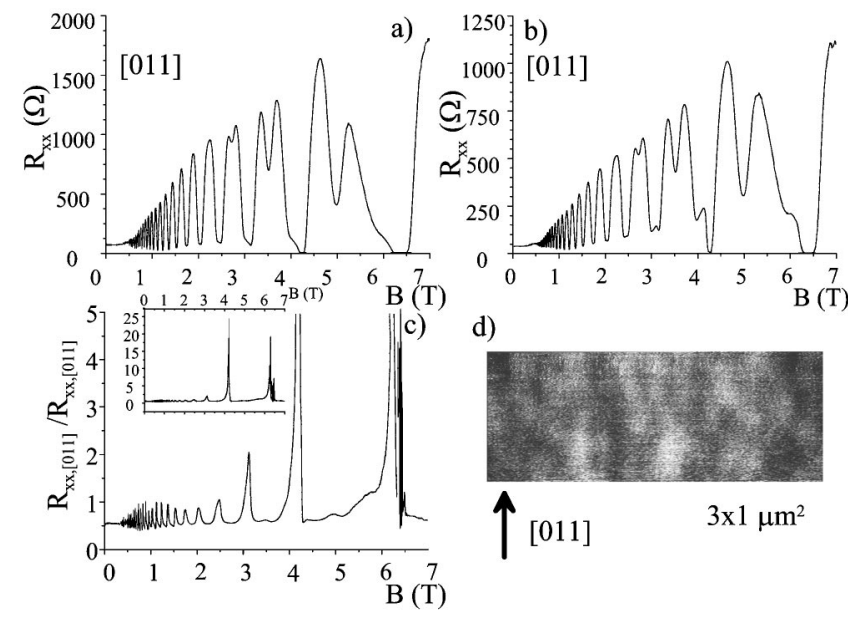

FIG. 5. (a) and (b): Resistance traces as a function of a magnetic field for Hall-bar samples, from the same wafer as the Corbino sample, oriented along [011], (a), and [011]], (b). (c): Ratio of (a) and (b). Inset: same on a different scale. (d) AFM image of surface morphology.

a fourfold symmetry as well. Instead, the twofold symmetry of Fig. 2(c) points to a surface-related anisotropy, which leads to anisotropic epitaxial growth. ${ }^{19}$ Very recently, it has been shown from atomic force microscopy (AFM) studies of the surface morphology that such anisotropy exists even for (Al)GaAs heterostructures containing 2DEG's with extremely high mobilities up to $2700 \mathrm{~m}^{2} / \mathrm{V} \mathrm{s}^{20}$ The magnetoresistance of the 2DEG's in these samples is extremely anisotropic in the fractional QHE-regime ${ }^{20}$. The surface morphology of our wafer is shown in Fig. 5(d) and is consistent with the data of Ref. 20. A surface corrugation of a few nanometers height and $\mathrm{a} \sim 1 \mu \mathrm{m}$ period is clearly discernible with ridges preferentially in the high mobility [011] direction $^{21}$. Associated with the ridges are density modulations, assumed to be of the order of a few percent at most. $^{22,20}$

Direct measurements of the electrical anisotropy were made by making Hall bars along the [011] and [011] directions. In the dark, the zero-field mobility was isotropic (within the accuracy of a few percent), while some anisotropy developed at high magnetic fields. After illumination with white light, the anisotropy strongly increased, presumably coincident with the second subband occupation near 5 $\times 10^{15}$ electrons $/ \mathrm{cm}^{2}$. Our anisotropies are comparable to or somewhat larger than those typically observed for similar samples. ${ }^{23}$ Their origins and the relation to the second subband occupation are still under discussion. ${ }^{23}$

Magnetoresistance traces for the two directions are shown in Figs. 5(a) and 5(b)). To emphasize the anisotropy, the ratio of the two tracks is displayed in Fig. 5(c). At modest magnetic fields, the anisotropy ratio fluctuates around the zerofield value, but explodes in the regime of the second subband occupation. In these field ranges, the electrons in the lowest subband are localized, so that transport is governed by the second subband only. The Corbino thermopower [Fig. 1(b)] qualitatively follows the field, dependence of the transport anisotropy [Fig. 5(c)]. Note that at high magnetic fields, the second subband is populated only in narrow field ranges, as follows from Fig. 1(d). It is thus concluded that the Corbino magnetothermopower is strongly coupled to the anisotropic transport properties of the sample. The light-induced high electron density makes our samples particularly well suited to reveal the correlation between thermopower and sample homogeneity, because of the relatively strong anisotropy. This is especially true under the condition that only the second subband accounts for the total transport. Our observations also explain why at least $S_{x y}$ is very material dependent as reported previously. ${ }^{2}$ For instance, a sign change, such as discussed in Figs. 4(b) and 4(d), is present in one sample, but not in another. ${ }^{2}$

As has been shown theoretically, intrinsic electron and phonon anisotropies may cause a finite $\epsilon_{x x} \cdot{ }^{3}$ On theoretical grounds it was also concluded that the derivative relations ${ }^{1}$ can be explained in terms of density inhomogeneities. ${ }^{5} \mathrm{We}$ have presented direct experimental evidence that MBEgrowth related anisotropic inhomogeneities dominate the Corbino magnetothermopower, and along with this the NE effect.

We would like to acknowledge W.C. van der Vleuten and P.A.M. Nouwens for the sample growth and preparations, and D.M. Bruls for the AFM measurements.
${ }^{1}$ B. Tieke, R. Fletcher, U. Zeitler, A.K. Geim, M. Henini, and J.C. Maan, Phys. Rev. Lett. 78, 4621 (1997).

${ }^{2}$ T.M. Fromhold, P.N. Butcher, G. Qin, B.G. Mulimani, J. P Oxley, and B.L. Gallagher, Phys. Rev. B 48, 5326 (1993).

${ }^{3}$ P.N. Butcher and M. Tsaousidou, Phys. Rev. Lett. 80, 1718 (1998).

${ }^{4}$ R. Fletcher, J.C. Maan, K. Ploog, and G. Weimann, Phys. Rev. B 33, 7122 (1986).

${ }^{5}$ S.H. Simon and N.R. Cooper, Phys. Rev. B 56, R7116 (1997).

${ }^{6}$ A.M. Chang and D.C. Tsui, Solid State Commun. 56, 153 (1985).

${ }^{7}$ S.H. Simon and B.I. Halperin, Phys. Rev. Lett. 73, 3278 (1994); I.M. Ruzin, N.R. Cooper, and B.I. Halperin, Phys. Rev. B 53, 1558 (1996).
${ }^{8}$ A.C. Beer, J.A. Armstrong, and I.N. Greenberg, Phys. Rev. 107, 1506 (1957).

${ }^{9}$ J.P. Heremans, C.M. Thrush, and D.T. Morelli, Phys. Rev. Lett. 86, 2098 (2001); J.P. Heremans, C.M. Thrush, and D.T. Morelli, Phys. Rev. B 65, 035209 (2002).

${ }^{10}$ Properties of GaAs, 3rd edition, edited by M.R. Brozel and G.E. Stillman (INSPEC, London, 1996), p. 28.

${ }^{11}$ R.O. Carlson, G.A. Slack, and S.J. Silverman, J. Appl. Phys. 36, 505 (1965).

${ }^{12}$ R.M. Kusters, J. Singleton, G. Gobsch, G. Paasch, D. Schulze, F.A. Wittekamp, G.A.C. Jones, J.E.F. Frost, D.C. Peacock, and D.A. Ritchie, Superlattices Microstruct. 9, 55 (1991).

${ }^{13}$ R.J.F. van Haren, F.A.P. Blom, and J.H. Wolter, Phys. Rev. Lett. 74, 1198 (1995). 
${ }^{14}$ H. van Zalinge, B. Özyilmaz, A. Böhm, R.W. van der Heijden, J.H. Wolter, and P. Wyder, Phys. Rev. B 64, 235303 (2001).

${ }^{15}$ H.S. Carslaw and J.C. Jaeger, Conduction of Heat in Solids (Clarendon Press, Oxford, 1959). Eq. (4.6.19).

${ }^{16}$ G.L. Pollack, Rev. Mod. Phys. 41, 48 (1969).

${ }^{17}$ H. Oji, J. Phys. C 17, 3059 (1984).

${ }^{18}$ B. Tieke, R. Fletcher, U. Zeitler, M. Henini, and J.C. Maan, Phys. Rev. B 58, 2017 (1998).

${ }^{19}$ A. Ballestad, B.J. Ruck, M. Adamcyk, T. Pinnington, and T.
Tiedje, Phys. Rev. Lett. 86, 2377 (2001), and references herein. ${ }^{20}$ R.L. Willett, J.W.P. Hsu, D. Natelson, K.W. West, and L.N. Pfeiffer, Phys. Rev. Lett. 87, 126803 (2001).

${ }^{21}$ Y. Tokura, T. Saku, S. Tarucha, and Y. Horikoshi, Phys. Rev. B 46, R15 558 (1992).

${ }^{22}$ J.H. Smet, K. von Klitzing, D. Weiss, and W. Wegscheider, Phys. Rev. Lett. 80, 4538 (1998).

${ }^{23}$ D. Reuter, M. Versen, M.D. Schneider, and A.D. Wieck, J. Appl. Phys. 88, 321 (2001). 